\title{
New Method of Predicting the Thermodynamic Properties of Solutions
}

\author{
Suntsov Yuri Konstantinovich* \\ Department of Physical and Analytical Chemistry, Voronezh State University of Engineering Technologies, Voronezh 394000, Russia
}

Received: August 03, 2013 / Accepted: August 28, 2013 / Published: March 25, 2014.

\begin{abstract}
Thermodynamic functions solutions of a 25 binary systems formed $n$-alcohols and esters of aliphatic acids by were calculated using the standards ideal solution and ideal gas. The value change regularity of the thermodynamic functions of solutions depending on their molar mass and concentration of the solutions' components was determined by the thermodynamic analysis. The method of prediction of the thermodynamic properties of binary solutions was suggested on the basis of the determined regularities. The corresponding equations were obtained.
\end{abstract}

Key words: Vapor-liquid equilibrium, Helmholtz energy, enthalpy, entropy of solutions, equation of state.

\section{Introduction}

Thermodynamic properties of solutions are a basis for calculations of the technological parameters, defining a direction and speed of technological processes. In the USA, it is counted up, that the economy of energy on distillation processes conducts $10 \%$ to annual economy of 500 million dollars [1]. Calculation of thermodynamic properties of solutions in full volume is practically realizable only on the basis of experimental data about phase equilibriums of various types. However, research of phase equilibriums is connected with the big experimental difficulties. In connection with this, the authors understand the importance of prediction of thermodynamic properties of solutions, based on the minimum number of experimental data. The solution to this problem is connected with the establishment of relationships between thermodynamic properties of solutions and properties forming their components. Data on the properties of binary systems containing members of a homologous series are of great interest

\footnotetext{
*Corresponding author: Suntsov Yuri Konstantinovich, Dr., research fields: theoretical and experimental studies of phase equilibriums and thermodynamic properties of solutions. E-mail: yurisuncov@yandex.ru.
}

in establishing relations between the molecular characteristics of components and the thermodynamic characteristics of solutions. The existing methods for calculating the properties of multi component systems are also based on the properties of their binary constituents [1]. Analysis of the scientific literature shows that the properties of these solutions are fragmented [2]. This required from us conducting additional research of large. The liquid-vapor equilibrium for 33 binary systems and also their volume and thermodynamic properties were studied previously, for example [3-5]. In this work, an attempt was made to determine the relation between the contributions of intermolecular interactions to the thermodynamic functions of binary solutions and the molecular weights (structure) and the concentration of solution components. The paper discusses the solutions of 25 binary systems formed: (1) Ethanol (a common solvent) and methyl ethanoate, ethyl ethanoate, $n$-propyl ethanoate, $n$-butyl ethanoate and $n$-pentyl ethanoate (the second solution components); (2) Ethanol (a common solvent) and methyl propanoate, ethyl propanoate, $n$-propyl propanoate, $n$-butyl propanoate and $n$-pentyl propanoate (the second solution components); (3) Ethanol (a common 
solvent) and methyl butanoate, ethyl butanoate, $n$-propyl butanoate, $n$-butyl butanoate and $n$-pentyl butanoate (the second solution components); (4) $n$-Propanol (a common solvent) and methyl ethanoate, ethyl ethanoate, $n$-propyl ethanoate, $n$-butyl ethanoate and $n$-pentyl ethanoate (the second solution components); (5) n-Butanol (a common solvent) and methyl ethanoate, ethyl ethanoate, $n$-propyl ethanoate, $n$-butyl ethanoate and $n$-pentyl ethanoate (the second solution components).

\section{Results and Discussion}

The $p-x-y-T$ data obtained were used in the well known thermodynamic equations [1] to calculate the activity coefficients $\left(\gamma_{i}\right)$, excess component chemical potentials in mixtures $\left(\mu_{i}\right)$, and excess Gibbs energies $\left(G^{e}\right)$ of solutions at $p, T=$ const. The excess entropies of solutions were calculated using Eq. (1).

$$
S^{e}=-\left(\partial G^{e} / \partial T\right)_{p, x_{1}, \cdots x_{k-1}}
$$

While the excess enthalpies of solutions were calculated using the Gibbs equation. Concentration and temperature dependences of the solution properties are indicative of a complex character of molecular interactions in the solutions, which manifests itself in deviations of binary systems from ideality (Fig. 1 and Table 1).

Lewis-Randal calculations do not allow us to directly relate the thermodynamic function values of solutions to the molecular weight (structure) of solution components. The chief disadvantage of calculations by the Lewis-Randal method is that thermodynamic functions of solutions are not related directly to molecular characteristics of components of mixtures [6, 7]. To achieve the assigned tasks the following algorithm of research was used: (1) Thermodynamic properties of liquid substances in homologous series were investigated; (2) Properties of solutions of the binary liquid systems formed by the common solvent and substances of these homologous series were investigated; (3) An ideal gas at the temperature $(T)$, volume $(V)$ and composition $(x)$ of the real liquid, which obeyed the statistics of the real liquid, was used as a standard for calculating the contributions of intermolecular interactions to the thermodynamic functions of solutions; (4) For the solutions at a constants molar concentration (at $T, V=$ const.), the functional relationship between the thermodynamic properties of solutions and the molar mass (structure) of the components forming solutions was attempted to be determined [8, 9].

An ideal gas has two degrees of freedom; therefore, the authors shall impose two restrictions. First, it is necessary to exclude work against any forces, except for those of intermolecular interaction therefore $V=$ const. Consider two possible cases: (1) the adiabatic case, $S=$ const. (2) the isothermal case ( $T=$ const.) -when the entropy of the system varies due to the intermolecular interaction rather than temperature. These reasons explain the necessity of conducting the process under condition of $V, T=$ const.. The intermolecular interaction to the thermodynamic function $\left(F^{r}\right)$ is defined as:

$$
F^{r}=F^{*}-F
$$

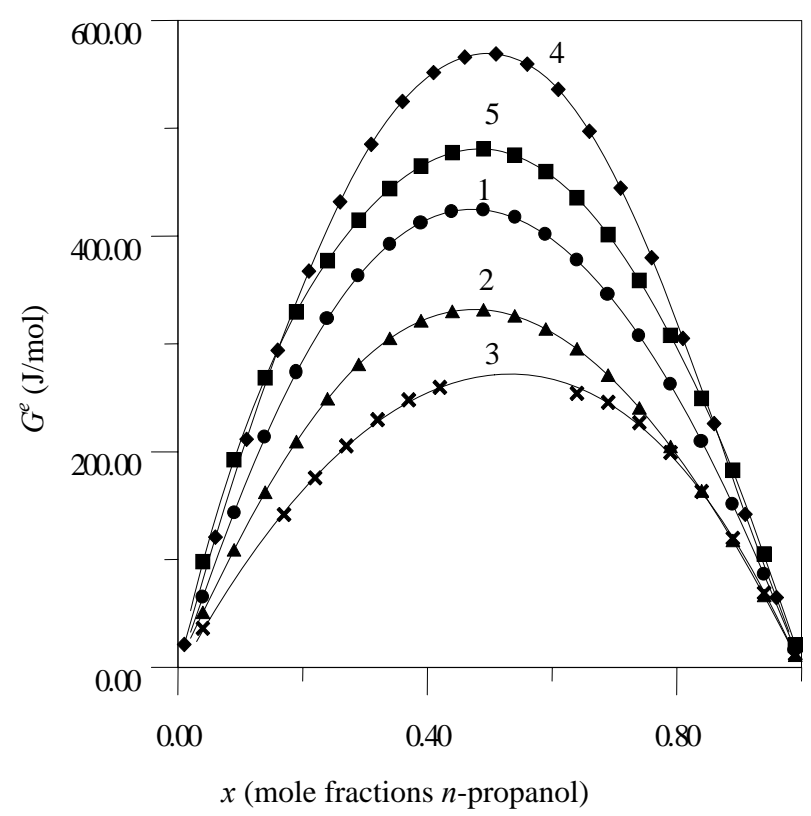

Fig. 1 Dependences of excess Gibbs energy $G^{e}$ on the composition of: 1-n-propanol-methyl ethanoate; 2-n-propanol-ethyl ethanoate; 3-n-propanol-n-propyl ethanoate; 4-n-propanol-n-butyl ethanoate; 5 - n-propanol-n-pentyl ethanoate; $T=353.15 \mathrm{~K}$. 
Table 1 Vapor-liquid equilibrium and thermodynamic properties of the binary system.

\begin{tabular}{|c|c|c|c|c|c|c|c|}
\hline $\begin{array}{l}X \text { (mole fractions } \\
n \text {-butanol) }\end{array}$ & $\begin{array}{l}Y \text { (mole fractions } \\
n \text {-butanol) }\end{array}$ & $\begin{array}{l}P \times 10^{5} \\
\left(\mathrm{~N} / \mathrm{m}^{2}(\mathrm{~Pa})\right)\end{array}$ & $\begin{array}{l}V \times 10^{-5} \\
\left(\mathrm{~m}^{3} \cdot \mathrm{mol}^{-1}\right)\end{array}$ & $\begin{array}{l}A^{r} \times 10^{4}\left(\mathrm{~J} \cdot \mathrm{mol}^{-1}\right) \\
\text { Helmholtz energy }\end{array}$ & $\begin{array}{l}H \times 10^{4} \\
\left(\mathrm{~J} \cdot \mathrm{mol}^{-1}\right)\end{array}$ & $\begin{array}{l}U^{r} \times 10^{4} \\
\left(\mathrm{~J} \cdot \mathrm{mol}^{-1}\right)\end{array}$ & $\begin{array}{l}G^{e} \\
\left(\mathrm{~J} \cdot \mathrm{mol}^{-1}\right)\end{array}$ \\
\hline \multicolumn{8}{|c|}{$n$-butanol-methyl ethanoate $T(\mathrm{~K})=353 \mathrm{~K}$} \\
\hline 1.0000 & 1.0000 & 21,291 & 9.7129 & 18,378 & 47,295 & 44,361 & 0 \\
\hline 0.9000 & 0.3770 & 52,315 & 9.5868 & 17,537 & 47,889 & 44,958 & 197 \\
\hline 0.8000 & 0.2236 & 79,232 & 9.4718 & 16,789 & 48,118 & 45,190 & 301 \\
\hline 0.7000 & 0.1534 & 102,990 & 9.3569 & 16,063 & 48,026 & 45,099 & 383 \\
\hline 0.6000 & 0.1125 & 123,988 & 9.2420 & 15,361 & 47,887 & 44,962 & 441 \\
\hline 0.5000 & 0.0850 & 142,692 & 9.1270 & 14,687 & 47,642 & 44,719 & 473 \\
\hline 0.4000 & 0.0646 & 159,517 & 9.0121 & 14,042 & 47,164 & 44,243 & 475 \\
\hline 0.3000 & 0.0480 & 174,903 & 8.8972 & 13,432 & 45,990 & 43,069 & 443 \\
\hline 0.2000 & 0.0333 & 189,261 & 8.7822 & 12,865 & 43,547 & 40,627 & 369 \\
\hline 0.1000 & 0.0182 & 203,713 & 8.6673 & 12,349 & 39,015 & 36,096 & 243 \\
\hline 0.0000 & 0.0000 & 215,605 & 8.5438 & 11,973 & 29,849 & 26,931 & 0 \\
\hline \multicolumn{8}{|c|}{$n$-butanol-ethyl ethanoate $T(\mathrm{~K})=353 \mathrm{~K}$} \\
\hline 1.0000 & 1.0000 & 21,291 & 9.7129 & 18,378 & 47,295 & 44,361 & 0 \\
\hline 0.9000 & 0.5618 & 34,530 & 9.8206 & 17,735 & 46,350 & 43,417 & 133 \\
\hline 0.8000 & 0.3761 & 46,262 & 9.9151 & 17,141 & 45,240 & 42,309 & 223 \\
\hline 0.7000 & 0.2713 & 56,928 & 10.010 & 16,566 & 44,044 & 41,114 & 295 \\
\hline 0.6000 & 0.2067 & 65,993 & 10.104 & 16,011 & 42,660 & 39,731 & 346 \\
\hline 0.5000 & 0.1646 & 73,126 & 10.199 & 15,487 & 41,102 & 38,173 & 368 \\
\hline 0.4000 & 0.1278 & 79,992 & 10.293 & 15,000 & 39,404 & 36,476 & 352 \\
\hline 0.3000 & 0.0948 & 86,658 & 10.388 & 14,543 & & & \\
\hline 0.2000 & 0.0634 & 93,324 & 10.482 & 14,117 & 35,805 & 32,878 & 231 \\
\hline 0.1000 & 0.0316 & 100,323 & 10.577 & 13,719 & 34,158 & 31,232 & 127 \\
\hline 0.0000 & 0.0000 & 107,523 & 10.661 & 13,359 & 32,845 & 29,920 & 0 \\
\hline \multicolumn{8}{|c|}{$n$-butanol—n-propyl ethanoate $T(\mathrm{~K})=353 \mathrm{~K}$} \\
\hline 1.0000 & 1.0000 & 21,291 & 9.7129 & 18,378 & 47,295 & 44,361 & 0 \\
\hline 0.9000 & 0.7563 & 26,797 & 10.002 & 17,812 & 47,398 & 44,464 & 235 \\
\hline 0.8000 & 0.5846 & 30,930 & 10.274 & 17,422 & 46,852 & 43,919 & 305 \\
\hline 0.7000 & 0.4613 & 34,663 & 10.546 & 17,044 & 46,078 & 43,145 & 364 \\
\hline 0.6000 & 0.3597 & 38,396 & 10.819 & 16,677 & 45,477 & 42,545 & 415 \\
\hline 0.5000 & 0.2995 & 40,796 & 11.091 & 16,334 & 45,175 & 42,244 & 442 \\
\hline 0.4000 & 0.2469 & 42,796 & 11.363 & 16,031 & 45,151 & 42,220 & 432 \\
\hline 0.3000 & 0.1907 & 44,662 & 11.635 & 15,771 & 45,133 & 42,202 & 381 \\
\hline 0.2000 & 0.1291 & 46,529 & 11.907 & 15,540 & 44,648 & 41,718 & 303 \\
\hline 0.1000 & 0.0684 & 48,129 & 12.180 & 15,337 & 43,032 & 40,101 & 197 \\
\hline 0.0000 & 0.0000 & 48,528 & 12.445 & 15,235 & 36,970 & 34,040 & 0 \\
\hline \multicolumn{8}{|c|}{$n$-butanol—n-butyl ethanoate $T(\mathrm{~K})=353 \mathrm{~K}$} \\
\hline 1.0000 & 1.0000 & 21,291 & 9.7439 & 18,378 & 47,295 & 44,361 & 0 \\
\hline 0.9000 & 0.8498 & 22,624 & 10.188 & 18,100 & 56,064 & 53,130 & 146 \\
\hline 0.8000 & 0.7310 & 23,664 & 10.632 & 17,850 & 63,602 & 60,669 & 273 \\
\hline 0.7000 & 0.6358 & 24,384 & 11.076 & 17,630 & 67,861 & 64,927 & 372 \\
\hline 0.6000 & 0.5595 & 24,811 & 11.519 & 17,450 & 67,264 & 64,331 & 441 \\
\hline 0.5000 & 0.4988 & 24,927 & 11.963 & 17,210 & 61,564 & 58,630 & 465 \\
\hline 0.4000 & 0.4361 & 24,758 & 12.407 & 17,210 & 51,833 & 48,899 & 437 \\
\hline 0.3000 & 0.3704 & 24,264 & 12.851 & 17,210 & 40,469 & 37,536 & 354 \\
\hline 0.2000 & 0.2738 & 23,491 & 13.295 & 17,210 & 31,195 & 28,261 & 249 \\
\hline 0.1000 & 0.1532 & 22,398 & 13.739 & 17,250 & 29,056 & 26,123 & 116 \\
\hline 0.0000 & 0.0000 & 21,265 & 14.183 & 17,280 & 39,580 & 36,647 & 0 \\
\hline
\end{tabular}


Table 1 continued

\begin{tabular}{llllllll}
\hline $\begin{array}{l}X \text { (mole fractions } \\
n \text {-butanol) }\end{array}$ & $\begin{array}{l}Y \text { (mole fractions } \\
n \text {-butanol) }\end{array}$ & $\begin{array}{l}P \times 10^{5} \\
\left(\mathrm{~N} / \mathrm{m}^{2}(\mathrm{~Pa})\right)\end{array}$ & $\begin{array}{l}V \times 10^{-5} \\
\left(\mathrm{~m}^{3} \cdot \mathrm{mol}^{-1}\right)\end{array}$ & $\begin{array}{l}A^{r} \times 10^{4}\left(\mathrm{~J} \cdot \mathrm{mol}^{-1}\right) \\
\text { Helmholtz energy }\end{array}$ & $\begin{array}{l}H \times 10^{4} \\
\left(\mathrm{~J} \cdot \mathrm{mol}^{-1}\right)\end{array}$ & $\begin{array}{l}U^{r} \times 10^{4} \\
\left(\mathrm{~J} \cdot \mathrm{mol}^{-1}\right)\end{array}$ & $\begin{array}{l}G^{e} \\
\left(\mathrm{~J} \cdot \mathrm{mol}^{-1}\right)\end{array}$ \\
\hline $\begin{array}{llllll}n \text {-butanol-n} \text {-pentyl ethanoate } T(\mathrm{~K})=353 \mathrm{~K} \\
1.0000\end{array}$ & 21,291 & 9.7439 & 18,378 & 47,295 & 44,365 & 0 \\
0.9000 & 1.0000 & 20,998 & 10.325 & 18,265 & 47,991 & 44,134 & 274 \\
0.8000 & 0.9363 & 20,131 & 10.942 & 18,235 & 48,354 & 43,830 & 443 \\
0.7000 & 0.8637 & 19,331 & 11.558 & 18,226 & 48,128 & 43,455 & 529 \\
0.6000 & 0.7972 & 18,531 & 12.175 & 18,239 & 47,369 & 43,008 & 546 \\
0.5000 & 0.7315 & 17,732 & 12.791 & 18,283 & 46,282 & 42,488 & 511 \\
0.4000 & 0.6693 & 16,932 & 13.408 & 18,449 & 45,083 & 41,896 & 438 \\
0.3000 & 0.6396 & 15,998 & 14.024 & 18,723 & 43,935 & 41,233 & 338 \\
0.2000 & 0.6126 & 14,799 & 14.640 & 18,751 & 42,986 & 40,497 & 222 \\
0.1000 & 0.4842 & 12,932 & 15.257 & 19,059 & 42,481 & 39,689 & 101 \\
0.0000 & 0.3662 & 8,866 & 15.873 & 19,507 & 43,020 & 38,809 & 0
\end{tabular}

where, $F$ is a thermodynamic function for the real liquid and $F^{*}$ is the corresponding thermodynamic function for the ideal gas. Subtraction $\left(F^{*}-F\right)$ provides positive values of $F^{r}$. Note that the activation of the intermolecular interaction in the liquid will change the sign of $F^{r}$. Consider a binary liquid solution at temperature $(T)$ in equilibrium with vapor at pressure of the saturated vapor p. Its molar Helmholtz energy $(A)$ is given by:

$$
A=x_{1} \mu_{1}+x_{2} \mu_{2}-p V
$$

where, $x_{1}$ and $x_{2}$ are the mole fractions and $\mu_{1}$ and $\mu_{2}$ are the chemical potentials of the species in solution; $p$-pressure of the saturated vapor; $V$-is the molar volume of the solution. Because absolute values of the Helmholtz energy are unknown, take as a reference state an ideal-gas mixture of the same composition as the liquid solution at the same temperature and with the same molar volume. This is a liquid-like state with the properties of an ideal gas [8]. The pressure in this reference state is then $P^{*}=R T / V$ and the molar Helmholtz energy is:

$$
A^{*}=x_{1} \mu_{1}^{*}+x_{2} \mu_{2}^{*}-R T
$$

where, $\mu_{1}{ }^{*}, \mu_{2}{ }^{*}$-chemical potentials of 1 and 2 components in the reference state of the mixture; $R$ - the universal gas constant. Define a residual molar Helmholtz energy $A^{r}$ as the difference between the reference value and the real value:

$$
A^{r}=A^{*}-A=x_{1}\left(\mu_{1}^{*}-\mu_{1}\right)+x_{2}\left(\mu_{2}^{*}-\mu_{2}\right)-R T+P V
$$

For the real saturated-liquid solution, the chemical potential may be expressed as:

$$
\mu_{i}=\Gamma_{i}(T)+R T \ln \hat{f}_{i}
$$

where, specific to species $i$, is a function of $T$ only, originating as an integration constant, and is the fugacity (not the fugacity coefficient) of species $i$ in solution. Note that the value in the liquid solutions is the same as in the equilibrium vapor phase. If the vapor phase is considered an ideal gas, then its value is the partial pressure $\left(y_{i} P\right)$. For ideal gas, we have:

$$
\mu_{i}^{*}=\Gamma_{i}(T)+R T \ln \left(x_{i} P^{*}\right)
$$

The product is the partial pressure of species $i$ in the ideal gas, where $i$ is that of the liquid solution. Because $P^{*}=R T / V$, Eq. (7) becomes:

$$
\mu_{i}^{*}=\Gamma_{i}(T)+R T \ln \frac{x_{i} R T}{V}
$$

Combination Eqs. (5), (6) and (8), after division by $R T$ (making the equation dimensionless):

$$
\frac{A^{r}}{R T}=x_{1} \ln \frac{x_{1} R T}{\hat{f}_{1} V}+x_{2} \ln \frac{x_{2} R T}{\hat{f}_{2} V}-1+\frac{p V}{R T}
$$

where, $\hat{\mathrm{f}}_{1}, \hat{\mathrm{f}}_{2}$-is the fugacity 1 and 2 component in solution. For pure species $i\left(x_{2}=0\right)$ as limited cases:

$$
\frac{A_{i}^{r}}{R T}=\ln \frac{R T}{\hat{f}_{i} V_{i}}-1+\frac{p_{i}^{0} V_{i}}{R T}
$$

Using the known thermodynamic relation, for entropy $\left(S^{r}\right)$ and internal energy $\left(U^{r}\right)$, it can be expressed: 


$$
\begin{aligned}
& S^{r}=S^{*}-S=-\left(\frac{\partial F^{r}}{\partial T}\right)_{V, X}= \\
& =\frac{H}{T}-R\left(x_{1} \ln \frac{x_{1} R T}{\hat{f}_{1} V}+x_{2} \ln \frac{x_{2} R T}{\hat{f}_{2} V}\right) \\
& U^{r}=U^{*}-U=-T^{2}\left(\frac{\partial\left(F^{r} / T\right)}{\partial T}\right)_{V, X}=H-R T+P V
\end{aligned}
$$

where, $H, V, P$-molar enthalpy of vaporization, molar volume and pressure of the saturated vapor for a solution of composition $x_{1}$ of molar fractions; $\hat{\mathrm{f}}_{1}, \hat{\mathrm{f}}_{2}$-is the fugacity of species 1 and 2 in solution.

\section{Homologous Series of Substances}

The values of $A^{r}, U^{r}$ and $T S^{r}$ were calculated by Eqs. (9)-(12) for liquid $n$-alkyl acetates, $n$-alkyl propionates, $n$-alkyl butyrate's, $n$-alcohols, $n$-alkanes, $n$-bromoalkanes, $n$-chloroalkanes, $n$-organic acids, $n$-alkyl amines, $n$-alkylarenes. An analysis of the calculation results suggests that, in homologous series, the functions $A^{r}, U^{r}$ and $T S^{r}$ depend linearly on the molecular mass:

$$
\begin{gathered}
U^{r}=k_{1} M+b_{1} \\
A^{r}=k_{2} M+b_{2} \\
T S^{r}=k_{3} M+b_{3}
\end{gathered}
$$

The coefficients of Eqs. (13)-(15) were fit by the least-squares method using the Curve Expert 1.3 software (Table 2). Eqs. (13)-(15) make it possible to calculate $A^{r}, U^{r}$, and $T S^{r}$ values for the other members of the homologous series. For example, for $n$-heptyl propionate we obtained (kJ/mol): $J /$ calc. $=47,777$, $\left(U_{\text {expt }}\right.$ =47,709), $F_{\text {calc }}=24,719,\left(F_{\text {expt }}=24,681\right)$, and $T S_{\text {calc }}^{r}=$ 23,051, $\left(T S^{r}{ }_{\text {expt }}=22,887\right)$. The observed regularities in the behavior of the $A^{r}, U^{r}$ and $T S^{r}$ functions associated with the $\mathrm{M}$ is in liquid compounds of homologous series can be used for further refinements of group models of solutions (UNTPAC, ASOG) [1]. The fact that $A^{r}, U^{r}$ and $T S^{r}$ depend linearly on the molecular mass of the compound in a homologous series suggests that the structures of the liquids are similar. Since $A^{r}$, a function most accurately determined in experiments, characterizes the intermolecular interaction in liquids, it is interesting to establish how this function is related to the molar volume $V$ and saturation vapor pressure $\mathrm{p}$ of liquid compounds in homologous series. A correlation analysis reveals that, within a homologous series, $\ln p$ and $V$ depend linearly on $A^{r}$ :

$$
\begin{gathered}
\ln p=k_{4} A^{r}+b_{4} \\
V=k_{5} A^{r}+b_{5}
\end{gathered}
$$

The coefficients of Eqs. (13)-(17) are listed in the Table 2. These equations describe $A^{r}$ within a homologous series with an accuracy of $\pm 50 \mathrm{~J} / \mathrm{mol}$. Eqs. (16) and (17) yield $p$ and $V$ values for the other members of the homologous series. For example, for $n$-heptyl acetate at $T=323.15 \mathrm{~K}$, we obtained: $p_{\text {calc }}=$

Table 2 Coefficients of Eqs. (13)-(17) for compounds in homologous series.

\begin{tabular}{llllllllllll}
\hline Homologous series & $T(\mathrm{~K})$ & $k_{I}$ & $b_{1}$ & $k_{2}$ & $b_{2}$ & $k_{3}$ & $b_{3}$ & $k_{4}\left(\times 10^{-4}\right)$ & $b_{4}$ & $k_{5}$ & $b_{5}$ \\
\hline$n$-Alkanes & 293 & 318.9 & 2,411 & 188.9 & $-1,514$ & 129.9 & 3,926 & -4.446 & 16.17 & $6.171 \times 10^{-9}$ & $3.919 \times 10^{-5}$ \\
$n$-Bromalkanes & 293 & 267.4 & 3,383 & 180.6 & 6,312 & 86.82 & 2,929 & -4.941 & 17.46 & $6.644 \times 10^{-9}$ & $-1.485 \times 10^{-5}$ \\
$n$-Chlor alkanes & 293 & 300.0 & 3,613 & 185.6 & 880.4 & 114.4 & 4,493 & -4.932 & 17.29 & $6.313 \times 10^{-9}$ & $1.670 \times 10^{-6}$ \\
$n$-Alkylarenes & 353 & 317.3 & 3,468 & 150.4 & 2,187 & 166.9 & 1,281 & -4.051 & 17.12 & $8.203 \times 10^{-9}$ & $-1.818 \times 10^{-5}$ \\
$n$-Organic acids & 293 & - & - & 142.0 & $1,429 \times 10^{4}$ & - & - & -5.365 & 19.51 & $8.617 \times 10^{-9}$ & $-1.398 \times 10^{-4}$ \\
$n$-Alkylamines & 293 & 207.6 & $16.69 \times 10^{4}$ & 139.6 & 5,692 & - & - & -5.484 & 18.12 & $8.752 \times 10^{-9}$ & $-3.980 \times 10^{-5}$ \\
$n$-Haloarenes & 343 & 91.69 & $2,513 \times 10^{4}$ & 59.17 & $1,208 \times 10^{4}$ & 32.52 & $13,054-3.668$ & 16.41 & $1.732 \times 10^{-9}$ & $7.417 \times 10^{-5}$ \\
$n$-Alkyl ethanoates & 323 & 272.6 & 7,690 & 125.5 & 4,105 & 146.5 & 3,632 & -4.577 & 17.40 & $9.935 \times 10^{-3}$ & -48.85 \\
$n$-Alkyl propanoates & 353 & 136.1 & 1,264 & 195.1 & $1,417 \times 10^{4}$ & 58.75 & $12,931-3.893$ & 16.68 & $9.143 \times 10^{-3}$ & -14.37 \\
$n$-Alkyl butanoates & 353 & 1,756 & 16,040 & 134.7 & 1,307 & 40.88 & $14,720-4.009$ & -16.88 & $9.473 \times 10^{-9}$ & -1.901
\end{tabular}


$547 \mathrm{~Pa},\left(p_{\text {expt }}=580 \mathrm{~Pa}\right)$ and $V_{\text {calc }}=190.0 \mathrm{~cm}^{3} / \mathrm{mol}\left(V_{\text {expt }}\right.$ $\left.=187.5 \mathrm{~cm}^{3} / \mathrm{mol}\right)$. For $n$-heptyl propionate at $T=$ $353.15 \mathrm{~K}, p_{\text {calc }}=1,177.11 \mathrm{~Pa}\left(p_{\text {expt }}=1,144.6 \mathrm{~Pa}\right)$ and $V_{\text {calc }}=211.2 \mathrm{~cm}^{3} / \mathrm{mol}\left(V_{\text {expt }}=210.97 \mathrm{~cm}^{3} / \mathrm{mol}\right)$. As the $A^{r}$ function associated with intermolecular interaction increases, it becomes more difficult for molecules to pass into the gas phase; as a result, the saturation vapor pressure decreases exponentially. Since the entropy of a liquid is smaller than that of the corresponding ideal gas ( $T, V=$ const.), the switching off of the intermolecular interaction should increase entropy, or at least leave it unchanged. An analysis of the available data demonstrates that the linear increase of $A^{r}$ is accompanied by the linear growth of $T S$ for the intermolecular interaction in liquid compounds of homologous series. In accordance with universally accepted principles, an increase in the function $S=S^{*}$ $S_{\text {liq }}$ is indicative of an increase in the molecular ordering of the liquid. If the structures of the liquids comprising a homologous series are similar, then an increase in the molecular size should cause a decrease in the density of the packing of the molecules in the liquid, in agreement with the linear increase of the molar volume of the liquids in homologous series [10].

\section{Solutions of the Binary Systems}

Eqs. (9)-(12) were used to calculate the Helmholtz energies $A^{r}$, internal energies $U^{r}$, and entropies $S^{r}$ of the systems (as an example, the values of the functions for systems Table 1). For the $n$-propanol- $n$-alkyl ethanoate solutions (as for the ethanol- $n$-alkyl ethanoate, ethanol- $n$-alkyl propanoates, ethanol- $n$-alkyl butanoate, and $n$-butanol- $n$-alkyl ethanoate solutions), the isotherms the $U^{r}=f(x)$ and $S^{r}$ $=f(x)$ behave similarly (Figs. 2 and 3 ).

For all systems solutions, $A^{r}$ of Helmholtz energies increases with the molar weight of ester correspondingly, the vapor pressure of the solutions decreases (Table 1, Fig. 4). The presence of a minimum in the isotherm $A^{r}=f(x)$ for the $n$-butanol-ethyl ethanoate system is indicative of the

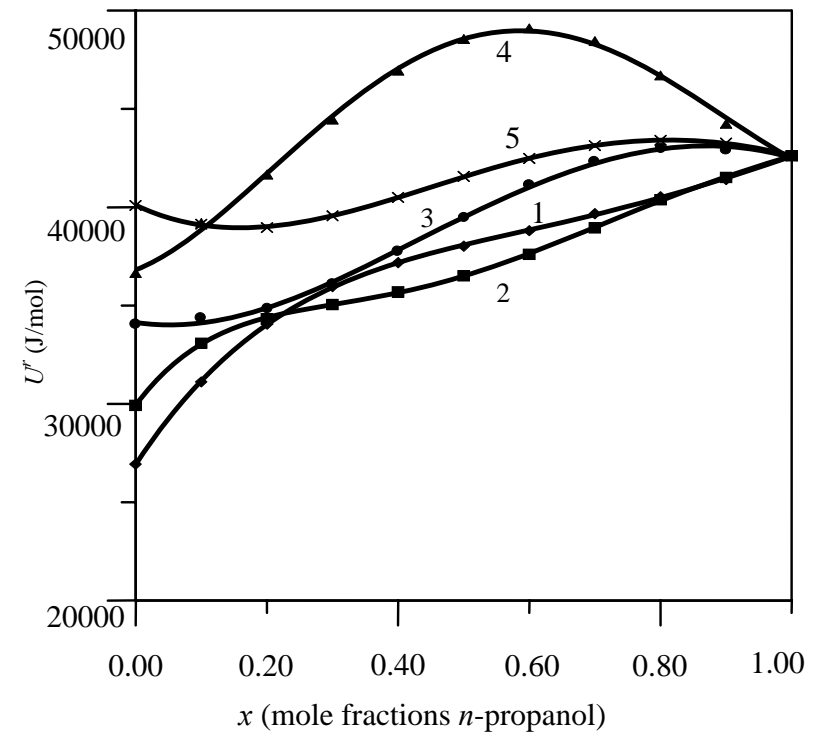

Fig. 2 Dependences of the internal energy $\left(U^{r}\right)$ of solutions on their composition at $353 \mathrm{~K}$ (Fig. 1 for notation).

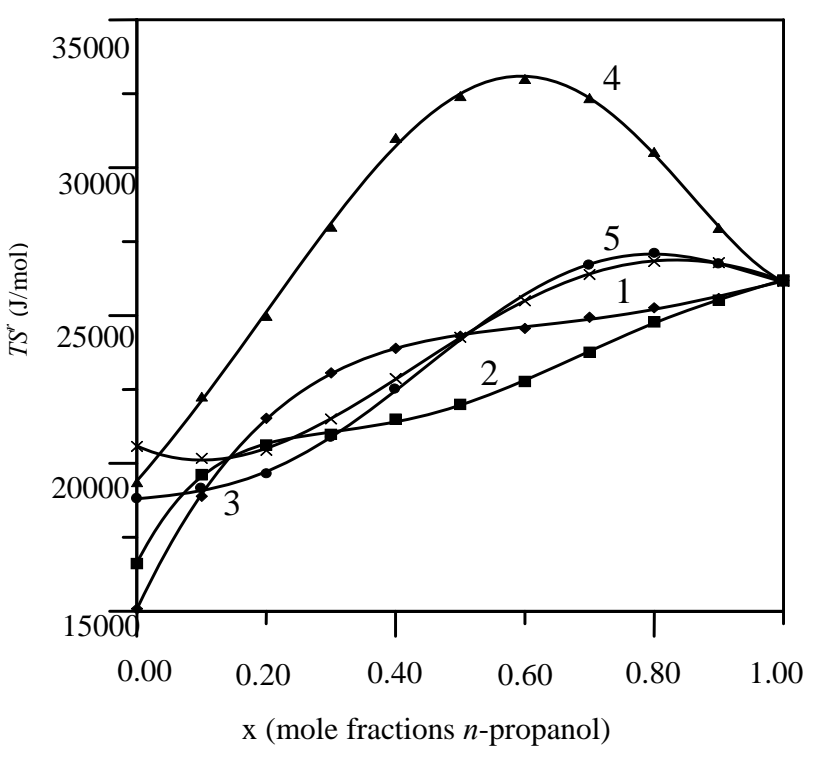

Fig. 3 Dependences of the entropy $T S^{r}$ of solutions on their composition at $353 \mathrm{~K}$ (Fig. 1 for notation).

most weak molecular interactions at this solution composition, which agrees with the existence of a positive azeotrope in this system (Table 1).

For solutions of constant molar concentrations, the Helmholtz energy was found to depend linearly on the molecular weight of substance in a homologous series, $A^{r}=k_{i} M+b_{i}$ (Fig. 5). The $k_{i}$ and $b_{i}$ coefficients of these isotherms in turn linearly depended on the composition of solutions. The results obtained allowed 


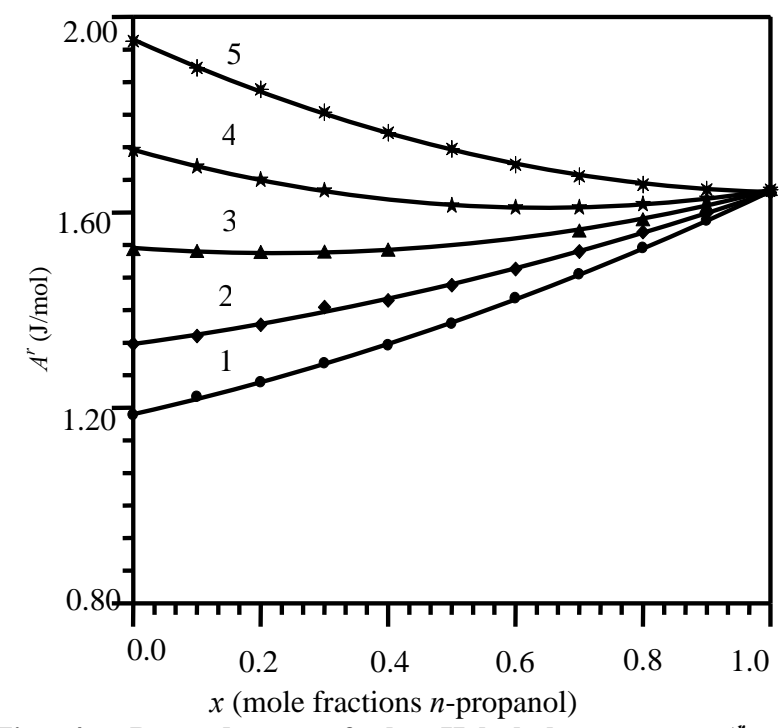

Fig. 4 Dependences of the Helmholtz energy $A^{r}$ of solutions on their composition at $343 \mathrm{~K}$ (Fig. 1 for notation).

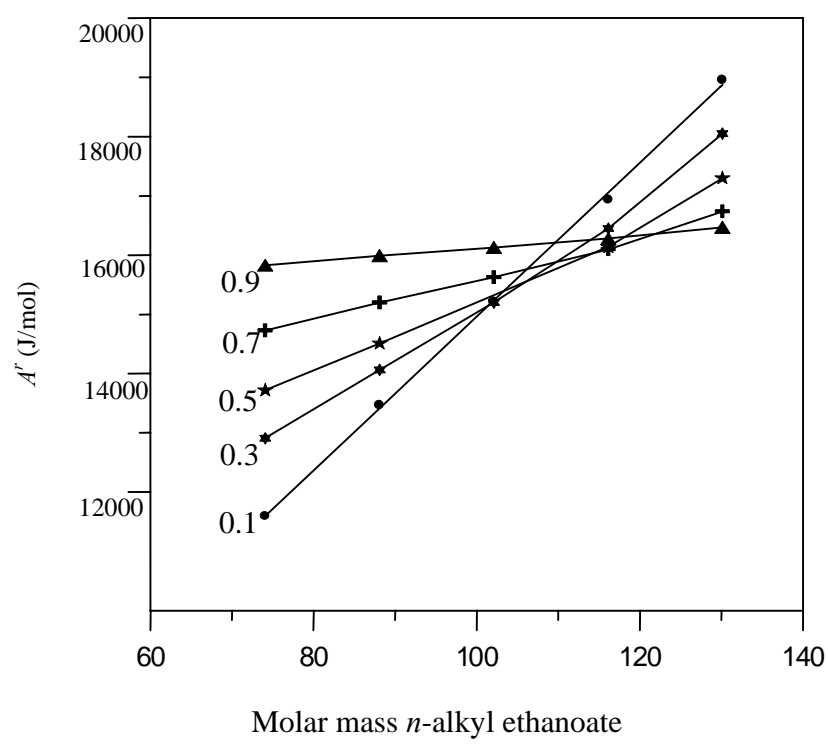

Fig. 5 Dependences of the Helmholtz energy $A^{r}$ of solutions of constant molar concentrations on the molecular weight of $n$-alkyl ethanoate in solutions for systems propanol-1-n-alkyl ethanoates; $T=353.15 \mathrm{~K}$ : 0.1 ; 0.3 ; 0.5 ; 0.7 ; 0.9 - constant mole fraction $n$-propanol in solutions of systems.

us to suggest Eq. (16).

$$
A^{r}=\left(k_{6} X+b_{6}\right) M+k_{7} X+b_{7}
$$

where, $X$ is the mole fraction of common solvent in the system and $M$ is the molecular weight of the second solution component Table 3 .

Eq. (16) describes $A^{r}$ accurate to $\sim 40 \mathrm{~J} / \mathrm{mol}$ and can be used for calculating properties of binary systems formed by $n$-alcohol and esters of aliphatic acids. The form of Eq. (16) is retained also at other temperatures. The following experimental data are necessary to find the factors in the Eq. (16) (at $T=$ const.) for two solutions of identical mole concentration, formed by the common solvent and two substances of homologous series: pressure of the saturated vapor $(P)$ composition of an equilibrium vapor phase $(Y)$, molar volume of a solution $(V)$. An accuracy prediction thermodynamic property of solutions depends only on the accuracy of the experimental data. The linear dependence of the Helmholtz energy for solutions of a constant molar composition on the molecular weight of the component in the homologous series (as with pure components, the boundary condition is $x_{1}=0$ ) can also be explained by the "similarity of the molecular-statistical structure of solutions" [10]. These results are in agreement with the hypothesis of additive contributions of chemical groups to the thermodynamic properties of solutions, which is basic for statistical models of solutions known as "group models" [1]. An equation similar to Eq. (16) was obtained for solutions of 33 binary systems formed by $n$-alcohols and compounds of the homologous series of esters of organic acids; formed by benzene (a common solvent) and methylbenzene, ethylbenzene, ethylbenzene, $n$-propylbenzene and $n$-butylbenzene (second solution components); formed by fluoroarene (a common solvent) and chloro-, bromo-, and iodoarenes (the second components) over the whole concentration range at various temperatures [11, 12].

The authors believe that this law is general in character and can be used to predict the thermodynamic properties of binary system solutions formed by a common solvent and representatives of homologous series of organic substances. Considering the widespread use in thermodynamic calculations by Lewis-Randall (standard ideal solution), it seemed appropriate to link this standard with the standard ideal gas. Define an excess dimensionless molar Helmholtz energy as: 
Table 3 Constants of the Eq. (16).

\begin{tabular}{lllllll}
\hline The name of systems & $T(\mathrm{~K})$ & $k_{6}$ & $b_{6}$ & $k_{7}$ & $b_{7}$ & ${\text { Accuracy }\left(\mathrm{J} \cdot \mathrm{mol}^{-1}\right)}^{-1}$ \\
\hline Ethanol— $n$-alkyl ethanoates & 323 & -117.54 & 119.38 & 11,045 & $4,566.9$ & 50 \\
Ethanol— $n$-alkyl propanoates & 353 & -136.37 & 132.91 & 12,766 & $1,520.5$ & 50 \\
Ethanol— $n$-alkyl butanoates & 353 & -152.56 & 141.93 & 14,925 & -132.03 & 20 \\
Propanol-1— $n$-alkyl ethanoates & 353 & -139.99 & 132.89 & 14,752 & 16,914 & 50 \\
Butanol-1— $n$-alkyl ethanoates & 353 & -136.39 & 133.92 & 166,666 & $1,552.4$ & 20 \\
Methyl ethanoate- $n$-alkyl ethanoates & 323 & -130.12 & 129.41 & $9,677.3$ & $3,578.1$ & 50 \\
Methyl propanoate- $n$-alkyl propanoates & 353 & -149.59 & 149.7 & $1,386.9$ & -432.77 & 50 \\
Methyl butanoate- $n$-alkyl butanoates & 353 & -152.7 & 141.9 & 14,925 & -132.0 & 20 \\
\hline
\end{tabular}

Table 4 The values of the excess Gibbs energy, calculated from the two standards: ideal solution and an ideal gas.

\begin{tabular}{lllll}
\hline$X($ mole & \multicolumn{3}{c}{$G^{e}(\mathrm{~J} / \mathrm{mol})$} \\
fractions & \multicolumn{1}{l}{ Ideal standard of solution } & Standard of an ideal gas & Ideal standard of solution & Standard of an ideal gas \\
\cline { 2 - 5 } n-butanol) & $n$-butanol—methyl ethanoate $T(\mathrm{~K})=353 \mathrm{~K}$ & $n$-butanol—n-pentyl ethanoate $T(\mathrm{~K})=353 \mathrm{~K}$ \\
\hline 1.00 & 0.0 & 0.0 & 0.0 & 0.0 \\
0.80 & 301 & 301 & 443 & 443 \\
0.60 & 441 & 441 & 546 & 546 \\
0.40 & 475 & 475 & 438 & 438 \\
0.20 & 369 & 369 & 222 & 222 \\
0.00 & 0.0 & 0.0 & 0.0 & 0.0 \\
\hline
\end{tabular}

$$
\frac{A^{e}}{R T} \equiv \frac{A^{r}}{R T}-x_{1} \frac{A_{1}^{r}}{R T}-x_{2} \frac{A_{2}^{r}}{R T}
$$

This equation, when combined with Eqs. (9) and (10) and after reduction yields:

$$
\begin{aligned}
& \frac{A^{e}}{R T}=x_{1} \ln \frac{1}{\gamma_{1}}+x_{2} \ln \frac{1}{\gamma_{2}}+x_{1} \ln \frac{V_{1}}{V}+ \\
& +x_{2} \ln \frac{V_{2}}{V}+\frac{p V-x_{1} p_{1} V_{1}-x_{2} p_{2} V_{2}}{R T}
\end{aligned}
$$

where, $\gamma_{1}, \gamma_{2}$-activity coefficients 1 and 2 component in solution; $V, p$-molar volume and pressure of the saturated vapor for a solution of composition $X_{1}$ of molar fractions; $p_{1}, p_{2}$-pressure of the saturated vapor $\left(\mathrm{N} / \mathrm{m}^{2}\right)$ and $V_{1}, V_{2}$-molar volume $\left(\mathrm{m}^{3} \cdot \mathrm{mol}^{-1}\right)$ of pure species 1 and 2, respectively. Eq. (18) connects the values of the excess energy of the Helmholtz solution and activity coefficients 1 and 2 component in solution. Next, using the well-known thermodynamic relations we can calculate the values of the chemical potentials, Gibbs energy, and all other thermodynamic functions. Values of the thermodynamics functions expected on the standard of ideal gas and ideal solution identical (for example
Table 4).

\section{Conclusions}

(1) Values of the Helmholtz energy $\left(A^{r}\right)$, internal energy $\left(U^{\prime}\right)$ and entropy $\left(T S^{\prime}\right)$ are linearly dependent on the molar mass of substance in homologous series;

(2) Increase of values of the Helmholtz energy $\left(A^{r}\right)$ for substances in homologous series is due to the linear magnification of molar volumes of fluid substances and reduction of pressure of the saturated vapor of substances under the exponential law;

(3) It is shown that the intermolecular interaction of components in solutions is expedient for estimating using the values of thermodynamic functions calculated under the standard of the ideal gas taken at the same temperatures and volume as the tested liquid and submitting to the same statistics. The correlation of thermodynamic properties of solutions with molecular characteristics of components forming them is determined provided that the solutions have constant mole concentration;

(4) It was determined by the analysis of thermodynamic properties of solutions of binary 
mixtures that Helmholtz energy $\left(A^{r}\right)$ of the binary solutions of the constant mole concentration formed by the common solvent and terms of homologous series of organic matters, are linearly dependent on molar mass of substance in homologous series. The equations describing thermodynamic properties of studied solutions with accuracy adequate for the technological purposes are obtained;

(5) The technique of predicting the thermodynamic properties of solutions of binary mixtures using properties of two pure components and two solutions of identical mole concentration is proposed. An accuracy prediction thermodynamic property of solutions depends only on the accuracy of the experimental data. Proposed a method speeds up the process of researching the thermodynamic properties of binary systems $\sim 340$ times.

\section{References}

[1] Walas, S. M. Phase Equilibria in Chemical Engineering; Butterworths: Boston, 1985.

[2] Vapor-Liquid Equilibrium Data. DDBST Dortmund Data Bank Software. Separation Technology GmbH. http://www.ddbst.com/ddb-vle.html.

[3] Suntsov, J.; Konyaev, B. Phase Equilibrium and Thermodynamic Properties of Binary Mixtures Ethanol-n-alkyl Propanoates. Russian Journal of Physical Chemistry 1999, 73(5), 710.
[4] Suntsov, J. Thermodynamic Functions of Liquid $n$-alkyl Propanoates and Their Binary Mixtures. Russian Journal of Physical Chemistry 2002, 76(5), 838.

[5] Suntsov, J.; Harchenko, G.; Zadorozhnyj, V. Liquid-Vapor Equilibrium in Systems $n$-butanol $n$-alkyl Ethanoates. Russian Journal of Physical Chemistry 2003, 77(1), 131.

[6] Rowlinson, J. B. Liquids and Liquid Mixtures; London, 1959.

[7] Rudakov, E. S. Molecular, Quantum and Evolutional Thermodynamics; Donetsk, 1998.

[8] Suntsov, Y. K. Change Regularities of Thermodynamic Properties of the Binary Mixtures Formed by Aliphatic Alcohol and Esters of Organic Acids. Dr. Thesis, Russian Chemistry and Technology University, 2004.

[9] Suntsov, J. In Legitimacies Change of Properties of Binary Systems on an Example of Solutions Formed by Aliphatic Alcohol and Complex Ethers of Organic Acids, Proceedings of 18 International Conference “Thermodynamics-2003”, Cambridge, Apr. 9-11, 2003.

[10] Suntsov, Y. K.; Goryunov, V. A. Correlation Dependences of the Thermodynamic Properties of Liquid Substituted Hydrocarbons Belonging to Various Homologous Series. Russian Journal of Physical Chemistry A. 2003, 77(12), 2073-2075.

[11] Suntsov, Y. K. Liquid-Vapor Phase Equilibria and the Thermodynamic Properties of Binary Solutions of n-alkylbenzenes. Russian Journal of Physical Chemistry A 2008, 82(4), 530-534.

[12] Suntsov, Y. K. Correlation Dependences of the Thermodynamic Properties of Liquid Haloarenes and Their Binary Solutions. Russian Journal of Physical Chemistry A 2008, 82(3), 332-336. 